\title{
Peri-Orbital Non-Invasive and Painless Skin Tightening-Safe and Highly Effective Use of Multisource Radio-Frequency Treatment Platform
}

\author{
Isabelle Rousseaux \\ Cabinet de Dermatologie Esthétique, Lille Côté Sud, Loos, France \\ Email: irousseaux@wanadoo.fr
}

Received 4 August 2015; accepted 5 September 2015; published 8 September 2015

Copyright (C 2015 by author and Scientific Research Publishing Inc.

This work is licensed under the Creative Commons Attribution International License (CC BY). http://creativecommons.org/licenses/by/4.0/

\section{(c) (i) Open Access}

\section{Abstract}

The periorbital area is the third highest-ranking area for cosmetic surgery. However, surgery in this area presents a number of difficulties and safety concerns. First generation Monopolar RF treatments in this area were usually associated with considerable pain and long downtime. In the present clinical study, we used the iFine handpiece of the EndyMed PRO, multi-source phase-controlled radiofrequency (RF) for Non-invasive, pain free, skin rejuvenation of the periorbital area. The study included eleven (11) subjects, treated for periorbital signs of aging (iFine handpiece, EndyMed PRO platform, EndyMed Medical, Caesarea, Israel). The degree of clinical improvement was assessed by the global aesthetic improvement scale (GAIS) and subjects satisfaction by post treatment questionnaires. $91 \%$ of patients showed good to excellent improvement as a result of the treatment. Subjects satisfaction showed that $55 \%$ of patients reported that they were very satisfied, $45 \%$ were satisfied while none were dissatisfied. There were no incidences of infections, scarring, hypopigmentation, or any other serious complications.

\section{Keywords}

Facial Tightening, Radiofrequency, EndyMed, 3DEEP ${ }^{\circledR}$, Periorbital

\section{Introduction}

The periorbital area is the third highest-ranking area for cosmetic surgery [1]. Blepharoplasty, eyelid surgery, has traditionally been used at a relatively early stage due to the limitations of non-surgical alternatives. However, 
surgery in this area presents a number of difficulties and safety concerns. In the present clinical study, we used the iFine handpiece of the Endymed PRO, multi-source phase-controlled radiofrequency (RF) for Non-invasive skin tightening around the eyes.

The periorbital area is one of the first areas to show the signs of ageing, laxity, fine lines and wrinkles. Treatment options are limited, due to the delicate nature of the skin in this area and safety concerns related to proximity to the eyeball. Blepharoplasty, whilst still being the third most common cosmetic surgical procedure, is not an option for many patients due to cost, risks associated with the general anesthesia, risks of scarring, and the post-operative recovery period [1]. Non-surgical options such as Botulinum toxin and dermal fillers can be effective, in the right hands, but also have inherent risks [2] [3]. Dermal fillers can lead to infection at the injection site as well as nodule formation or a bluish discoloration beneath the skin (the Tyndall phenomenon) due to superficial injection technique [2]. Complications of injecting Botulinum toxin include dry eye syndrome, which if unidentified can lead to eyelid swelling, epiphora (excessive tear production) and scleral show [3].

RF has been found to be effective in the safe delivery of energy into the skin, independent of skin color [4]. Resistance encountered by the RF energy flow causes a build-up of heat, which induces an immediate contraction of the collagen (an "instant lift") and stimulates a natural wound-healing response, production of new skin cells and collagen [6]. When focused in the dermis and hypodermis, non-ablative RF treatment can lead to improvements in the skin structure and tightening of lax and sagging skin. In addition to providing skin tightening, RF can be implemented for skin resurfacing and micro-needle dermal remodeling.

Due to the high safety profile of the treatment, it is suitable for most patient groups and all skin types [6]. Multiple RF technologies and devices are available, with varying levels of efficacy and safety [7].

Most RF devices use monopolar or bipolar technologies. Monopolar RF uses one RF generator and one electrode to deliver the RF energy into the skin, often using a grounding pad. Bipolar RF also uses one RF generator and the energy flows between two electrodes. Results with these technologies can be variable, patients are often exposed to high levels of energy, causes epidermal heating and discomfort, thus cooling must be implemented, and there are possible side effects including burns, purpura and hyperpigmentation [9]. Monopolar RF transmits RF from one electrode of the skin to a second electrode under the back. The flow of energy in this case in uncontrolled and may raise concerns especially when treating periorbital areas.

Multi-source phase-controlled RF is a sophisticated FDA-cleared RF technology that uses six RF generators and six electrodes. Proprietary software controls how the energy flows between the electrodes, with multiple fields of energy interacting and forcing the energy deep into the skin without overheating its surface. The result is a deep, volumetric heating of the dermis and hypodermis, which delivers high level and predictable clinical results with excellent patient comfort and a high safety profile [5]-[8].

Multi-source RF can also be implemented for fractional skin resurfacing, providing an epidermal and dermal skin rejuvenation effect. The multi-source RF micro-ablates up to $10 \%$ of the treated area with simultaneous volumetric heating of the dermis, resulting in good and predictable clinical outcomes [10] [11]. The downtime is shorter than traditional laser resurfacing procedures and the risk of side effects is minimal [10]. In comparison to monopolar technology in which the energy from the skin electrode to the back plate uncontrolled through the body, in the Multisource technology the emitting and return electrodes are in the handpiece and all energy will be confined to the skin .

Amy Patdu reported on her experience using multi-source RF for eye rejuvenation in Asian skin [4] about 19 patients who had completed a course of six treatments with the 3DEEP ${ }^{\circledR}$ iFine; a specially designed handpiece for the periorbital area that delivers heat to a depth of $2.8 \mathrm{~mm}$. During each treatment the skin temperature was raised to approximately $40^{\circ} \mathrm{C}$ and sustained for three minutes.

The technology allows pain-free, safe and effective treatment of the delicate and hard-to-reach skin immediately around the eyes and will reduce under eye bags, smooth and tighten the skin and lift the upper eyelid to reveal a more open eye. There is an immediate visible improvement, which disappears after one to two days but is an indication of the long-lasting result and is a great hook for first-time patients. More recently we have significantly enhanced our protocol and results for periorbital rejuvenation.

Inglefield [12] reported the combination of 2 different multisource RF treatments, combining a single treatment periorbital skin tightening and fractional skin resurfacing. The skin tightening treatment was carried out according to the standard protocol, then after application of a topical anesthetic the Fractional RF skin resurfacing (FSR) handpiece was used over the area. A course of four treatments spaced four weeks apart was performed. Inglefield found that the addition of the FSR, provided an additional tightening effect and epidermal ablation for 
a smoother, brighter result. Inglefield reported in his study overall patient satisfaction of $94 \%$ of patients very satisfied or extremely satisfied with the results they have achieved.

\section{Patients and Methods}

Eleven subjects (10 females, 1 male, ages of $61.1 \pm 8.6$ years) with periorbital signs of aging were enrolled in the study. All patients were Caucasians, Fitzpatrick's skin types 1 - 4. Inclusion criteria were visible signs of periorbital skin aging and more specifically visible peri-orbital wrinkles and or lax skin.

We excluded patients that have cardiac arrhythmia or pacemakers and patients that had undergone surgery or injection in treated area in the last 6 months.

Patients were photographed using standardized methods before each session. In each treatment session, each patient received a periorbital non-ablative skin tightening treatment. iFine handpiece of the EndyMed PRO treatment platform (Endymed Medical Ltd., Caesarea, Israel) was used (Figure 1). A total number of 6 sessions were performed for each patient. The treatment sessions were repeated every 2 weeks for the first four sessions, then once a month for the 2 last sessions.

Treated areas were visually assessed for skin responses, including edema, erythema, hypopigmentation, hyperpigmentation, and textural changes following the treatment. Global Aesthetic Improvement Scale (GAIS), validated by Day et al. [13], is a five grade scale where " 5 " is "very much improved" ( $75 \%-100 \%$ improvement), " 4 " is "much improved" ( $50 \%$ - $75 \%$ improvement), " 3 " is "improved" ( $25 \%$ - $50 \%$ improvement) 2 is minimal-no change ( $0 \%$ - $25 \%$ improvement) and " 1 " is "worse”. Patients' photographs were graded according to the Global Aesthetic Improvement Scale (GAIS) by a board certified dermatologist in addition to subjective satisfaction questionnaires filled by the patients.

Patients' photographs were graded according to the Global Aesthetic Improvement Scale (GAIS) by a board certified dermatologist in addition to subjective satisfaction of the patients.

\section{Results}

Six subjects had 6 treatment sessions, 1 had 8 sessions and 3 others had 10 sessions. Mild to moderate eryhema and mild edema were noted following the non-ablative Skin Tightening procedure. No unexpected adverse effects were detected or reported. There were no incidences of infections, scarring, hypopigmentation, or any other serious complications.

\section{Physician Evaluation}

All patients showed improvement. Excellent improvement ( $>76 \%$ ) was noted in 2 patients, very good improvement $(50 \%-75 \%)$ in 4 patients, good $(26 \%-50 \%)$ in 4 patients and minimal to no change in one patient (Figure 2, Figure 3, Table 1).
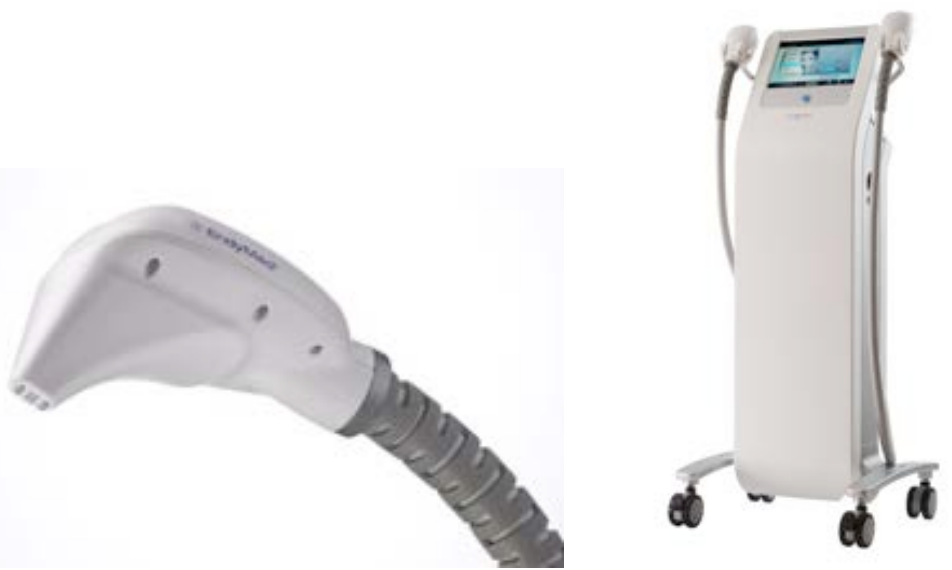

Figure 1. iFine Multisource RF for the periorbital and perioral areas. Rt. EndyMed Pro Multisource RF (3DEEP) treatment platform. 


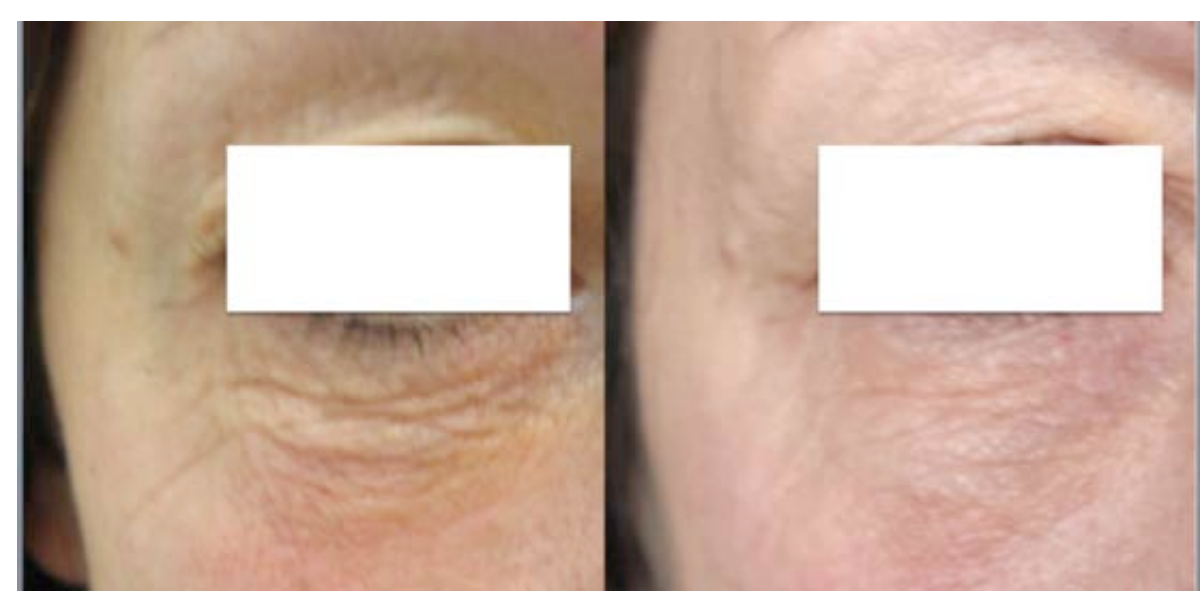

Figure 2. Before and after 6 sessions of EndyMed's iFine treatments. Significant improvement of texture, wrinkle reduction and tightening of the periorbital skin.

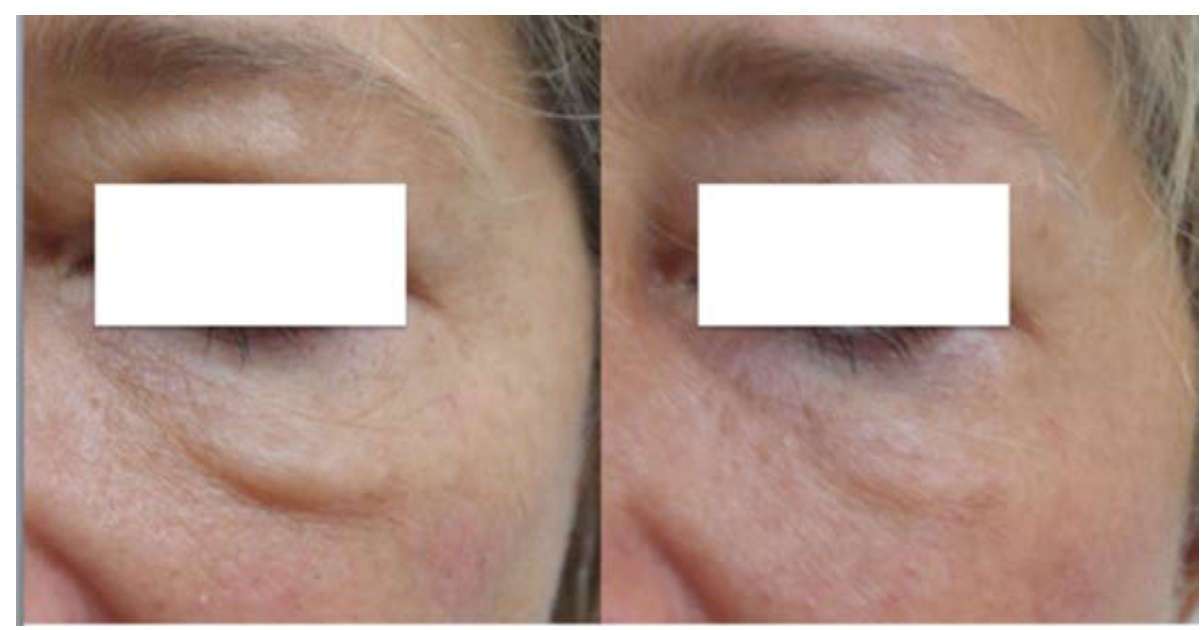

Figure 3. Before and after 4 sessions of EndyMed's iFine treatments. Significant improvement of texture, wrinkle reduction and tightening of the periorbital skin.

Table 1. Physician evaluation using the global aesthetic improvement scale (GAIS) showed improvement in $91 \%$ of patients.

\begin{tabular}{cccc}
\hline Global aesthetic improvement scale (GAIS) & Improvement & Patients & Percent \\
\hline Very much improved & & 2 & 37 \\
Much improved & $75 \%-100 \%$ & 4 & 36 \\
Improved & $50 \%-75 \%$ & 4 & 9 \\
Minimal to no change & $25 \%-50 \%$ & 1 & 0 \\
Worse & $0 \%-25 \%$ & 0 & \\
\hline
\end{tabular}

In addition to clinical evaluation of the results by the physician the patients were asked about their satisfaction with the treatment. The questionnaires suggested 5 grades of satisfaction. Very satisfied, satisfied, neutral, somewhat dissatisfied, and very dissatisfied. In our study, 55\% of patients reported that they were very satisfied, 45\% were satisfied while none were dissatisfied (Figure 4).

\section{Discussion and Conclusions}

Periorbital treatments were performed for the above study using multisource phase controlled system. iFine handpiece, used in this study, is specially designed to treat delicate areas such as periorbital and perioral. 


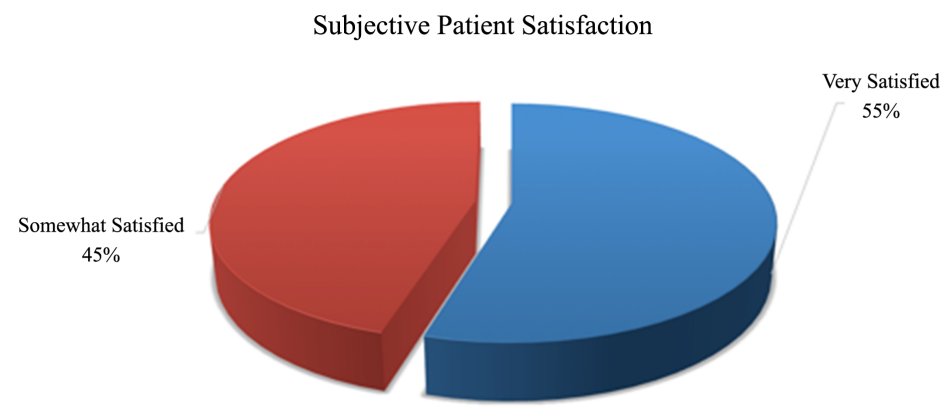

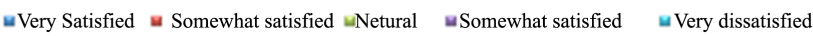

Figure 4. Patient Satisfaction questionnaires. When asked about treatment satisfaction $55 \%$ of patients reported that they were very satisfied, $45 \%$ were satisfied while none were dissatisfied.

Body and face non-ablative skin tightening using multisource radiofrequency (EndyMed PRO) is one of the leading technologies for this purpose in France. In this present report, we describe for the first time in France a clinical study of periorbital area, showing significant improvement of skin texture, skin laxity and eye bags.

Blepharoplasty and other periorbital surgical procedures have increased in popularity over the past few decades, notably including new solutions in skin tightening and rejuvenation and the treatment of facial rhytides [12]. However, lots of patients prefer non-invasive treatments with no to minimal downtime.

In our current study, Dermatologist's evaluation using the global aesthetic improvement scale (GAIS) showed good to excellent improvement in $91 \%$ of the patients and mild improvement in additional 9\%. Patients' subjective satisfaction was high.

The treatment was well accepted by the patients with minimal or no pain. There was no need for post therapy treatment and patients were able to return to work with makeup as soon as one hour after therapy.

Four to six weeks after treatment dermal changes were noted including significant improvement in skin texture, reduction of wrinkles and skin laxity.

Based on the above results, we believe the new EndyMed PRO iFine RF procedure provides an exciting new option for effective treatment of skin laxity around the eyes with minimal discomfort and downtime. To further explore this very promising technology there is a need for larger studies, with a larger range of skin types and longer follow up.

\section{References}

[1] American Society of Plastic Surgeons (2013) Plastic Surgery Statistics Report.

[2] Lafaille, P. and Benedetto, A. (2010) Fillers: Contraindications, Side Effects and Precautions. Journal of Cutaneous and Aesthetic Surgery, 3, 16-19. http://dx.doi.org/10.4103/0974-2077.63222

[3] Ozgur, O., Murariu, D., Parsa, A. and Parsa, F. (2012) Dry Eye Syndrome Due to Botulinum Toxin Type-A Injection: Guideline for Prevention. Hawai'i Journal of Medicine \& Public Health, 7, 120-123.

[4] Patdu, A. (2014) Non-Invasive Eye Rejuvenation of Asian Skin Using a Novel Multi-Source Phase-Controlled Radiofrequency Device. PRIME, 2, 19-27.

[5] Elman, M. and Harth, Y. (2011) Novel Multi-Source Phase-Controlled Radiofrequency Technology for Nonablative and Micro-Ablative Treatment of Wrinkles, Lax Skin and Acne Scars. Laser Therapy, 20, 139-144. http://dx.doi.org/10.5978/islsm.20.139

[6] Harth, Y. (2015) Painless, Safe, and Efficacious Noninvasive Skin Tightening Body Contouring, and Cellulite Reduction Using Multisource 3DEEP Radiofrequency. Journal of Cosmetic Dermatology, 14, 70-75. http://dx.doi.org/10.1111/jocd.12124

[7] De la Torre, J.R., Moreno-Moraga, J., Muñoz, E. and Navarro, P.C. (2011) Multisource, Phase-Controlled Radiofrequency for Treatment of Skin Laxity. Correlation between Clinical and In-Vivo Confocal Microscopy Results and Real-Time Thermal Changes. Journal of Clinical and Aesthetic Dermatology, 4, 28-35.

[8] Harth, Y. and Lischinsky, D. (2011) A Novel Method for Real-Time Skin Impedance Measurement during Radiofre- 
quency Skin Tightening Treatments. Journal of Cosmetic Dermatology, 10, 24-29. http://dx.doi.org/10.1111/j.1473-2165.2010.00535.x

[9] Paasch, U., Bodendorf, M.O., Grunewald, S. and Simon, J.C. (2009) Skin Rejuvenation by Radiofrequency Therapy: Methods, Effects and Risks. Journal der Deutschen Dermatologischen Gesellschaft, 7, 196-203. http://dx.doi.org/10.1111/j.1610-0387.2008.06780.x

[10] Dahan, S., Rousseaux, I. and Cartier, H. (2013) Multisource Radiofrequency for Fractional Skin Resurfacing-Significant Reduction of Wrinkles. Journal of Cosmetic \& Laser Therapy, 15, 91-97. http://dx.doi.org/10.3109/14764172.2012.748205

[11] Elman, M., Frank, I., Cohen-Froman, H. and Harth, Y. (2012) Effective Treatment of Atrophic and Icepick Acne Scars Using Deep Non-Ablative Radiofrequency and Multisource Fractional RF Skin Resurfacing. Journal of Cosmetics, Dermatological Sciences and Applications, 2, 267-272. http://dx.doi.org/10.4236/jcdsa.2012.24051

[12] Ingelfield, C. (2014) Radiofrequency Treatment for the Treatment of the Periorbital Region. Aesthetic Journal, 11, 45-46.

[13] Day, D.J., et al. (2004) The Wrinkle Severity Rating Scale: A Validation Study. American Journal of Clinical Dermatology, 5, 49-52. http://dx.doi.org/10.2165/00128071-200405010-00007 